\title{
Poststroke Fatigue Is Still a Neglected Issue: Findings from an Internet-Based Study on the Need for Information and Treatment in The Netherlands
}

\author{
Aglaia M. E. E. Zedlitz, ${ }^{1,2}$ Michelle van Eijk, ${ }^{3}$ Roy P. C. Kessels, ${ }^{1,4}$ \\ Alexander C. H. Geurts, ${ }^{5}$ and Luciano Fasotti ${ }^{1,6}$ \\ ${ }^{1}$ Donders Institute for Brain, Cognition and Behaviour, Radboud University Nijmegen, 6500 HE Nijmegen, The Netherlands \\ ${ }^{2}$ Department of Clinical Neuropsychology, Leiden University, 2300 RB Leiden, The Netherlands \\ ${ }^{3}$ G-Kracht Psychomedical Centre, 2611 KG, Delft, The Netherlands \\ ${ }^{4}$ Department of Medical Psychology, Radboud University Nijmegen Medical Centre, 6500 HB Nijmegen, The Netherlands \\ ${ }^{5}$ Department of Rehabilitation Medicine, Nijmegen Centre for Evidence Based Practice, Radboud University Nijmegen Medical Centre, \\ 6500 HB Nijmegen, The Netherlands \\ ${ }^{6}$ Research, Development and Education, Sint Maartenskliniek, 6500 GM Nijmegen, The Netherlands
}

Correspondence should be addressed to Roy P. C. Kessels, r.kessels@mps.umcn.nl

Received 16 March 2012; Accepted 29 April 2012

Academic Editors: J. Danckert, C. Fieschi, H. McNaughton, and A. Miller

Copyright $\odot 2012$ Aglaia M. E. E. Zedlitz et al. This is an open access article distributed under the Creative Commons Attribution License, which permits unrestricted use, distribution, and reproduction in any medium, provided the original work is properly cited.

Objective. To evaluate the need for information and treatment of poststroke fatigue (PSF) in community-dwelling patients with stroke in The Netherlands. Methods. Data were obtained from a Dutch internet-based questionnaire. The amount of information received about PSF, self-management and other treatment options, and the perceived need for treatment were scored. PSF was assessed with two Visual Numerical Scales of fatigue severity (VNSF), the Fatigue Severity Scale (FSS7), and by scoring the nature (physical versus mental) and onset time of PSF. Results. Data were available of 538 stroke survivors (mean age 52 years, $45 \%$ men, and mean time since onset 2.7 years). Severe fatigue (both VNSF items $\geq 7 ; 48 \%$ ) and severe fatigue impact (FSS7 $>5 ; 68 \%$ ) were frequent. Most participants (57\%) experienced both physical and mental fatigue. Only $17 \%$ of the respondents indicated to have had received sufficient information about PSF and 7\% about treatment options, whereas 56\% expressed a substantial need for treatment. The need for treatment was moderately associated with fatigue severity and fatigue impact, whereas information status was not related to any demographical, fatigue, or stroke characteristic. Conclusions. PSF is a significant symptom after stroke requiring medical attention and treatment. The results of this survey indicate that patients do not receive adequate information on PSF.

\section{Introduction}

Persisting fatigue is a common and debilitating consequence of stroke even in patients with good recovery [1-4]. Prevalence rates of poststroke fatigue (PSF) are substantial, varying between 38 and $73 \%$ [4]. These rates seem to be stable over time, with no marked decline after the postacute stage to even years following the injury [5-9]. As no unequivocal associations with clinical or demographical variables, such as stroke type, age, sex, or education, have been found, all patients who have experienced a stroke may be at risk to develop this symptom [4]. Furthermore, PSF has been found to have a negative effect on quality of life [2, 10], rehabilitation outcomes [11], and even mortality [1214]. However, PSF is often misdiagnosed $[11,15,16]$ and evidence-based clinical guidelines for dealing with PSF are scarce $[17,18]$.

The pathogenesis of PSF is still poorly understood $[4,12$, $19,20]$ but believed to be multifactorial $[4,5,12,14,20-$ 22]. Although direct associations with biological markers 
have been demonstrated only in a few studies [20], the brain damage itself is suggested to be a primary cause for the experienced fatigue [23-25]. Furthermore, associations with psychological distress such as depressive symptoms and anxiety have been repeatedly found [4]. The direction of this association has not been established, but somatic complaints seem to mediate this association [22]. Moreover, the consistent finding of double dissociations between these symptoms underscores the distinction between these syndromes $[3,22,26]$. However, psychological distress may certainly exacerbate fatigue and vice versa.

It has been accepted that PSF is a multifaceted phenomenon, including the severity of fatigue and the impact of fatigue on daily life [4]. Although no uniform definition of PSF exists, it is generally defined as "a subjective experience of extreme and persistent tiredness, weakness, or exhaustion after stroke, which can present itself mentally, physically or both and which is unrelated to previous exertion levels" $[18,27]$. Since this definition is intrinsically subjective and no derivative assessment tools have been specifically constructed for patients with stroke, a large variety of fatigue rating scales have been used in research and clinical practice [20, 28]. Some of these tools assess the dimension of fatigue severity, such as Visual Analogue Scales [29] or the Checklist Individual Strength [30]. Others, such as the Fatigue Severity Scale, measure the impact of fatigue in daily life [31]. The assessment of both aspects is needed to comprehensively assess PSF and to warrant the comparability of different studies.

A few qualitative studies in small focus groups have demonstrated that patients with PSF report a great need for information on the syndrome $[15,16]$. Patients reported that the lack of information led to confusion and distress, which in turn elongated the time needed to adjust to the fatigue [16]. They want to understand their experience of fatigue and have it diagnosed or explained so that they are less overwhelmed by it and know what to do about it [15]. Studies on the treatment of fatigue in patients with stroke and in patients with other neurological disease suggest that patient education, teaching energy conservation strategies, and graded activity training can be helpful in the management of fatigue $[18,32,33]$.

Despite the importance of education and treatment for patients with PSF, little research has been conducted to quantitatively identify the need for education and treatment possibilities amongst stroke survivors [17, 18]. Furthermore, PSF is often not accurately recognized $[11,15,16]$. The aim of the present study was to investigate the current provision of patient information and healthcare in The Netherlands, by an internet-based questionnaire, and to relate these needs to demographic, fatigue, and stroke characteristics.

\section{Methods}

Data were obtained from a Dutch internet-based questionnaire. External links to the website were placed on the Dutch Wikipedia site about stroke and on the website of The Netherlands Brain Foundation (Hersenstichting). All participants who spontaneously completed the questionnaire consented with its use for scientific research. No feedback about results or financial reward was given and data were anonymously collected.

To identify current information and need status, questions about the amount of information received with regard to (i) PSF, (ii) self-management techniques, and (iii) treatment had to be answered on a 5-point Likert Scale (ranging from none to ample information) as well was the need for treatment of PSF (from none to a great need). The sum of the information scores was used (range 0-15) to indicate information status.

A Visual Numerical Scale was used to assess the severity of fatigue (VNSF). The VNSF consisted of questions about perceived fatigue severity (ranging from 0 : no fatigue to 10 : extreme fatigue) at present (question 1) and over the past 2 weeks (question 2). A score above 6 on both VNSF items was regarded as severe fatigue [34]. The mean of both items was used for statistical analysis.

The Fatigue Severity Scale-7 (FSS7) [21] is a wellvalidated tool to assess fatigue impact. It contains the last 7 items of the original 9-item FSS [31], to be scored on a 7point Likert Scale. A mean score was calculated and a higher score than 5 indicated severe fatigue impact (range: 1-7) $[21,31]$.

Additional questions about the nature of PSF (physical versus mental) and postonset time of fatigue had to be answered on a 5-point Likert Scale. Furthermore, data were collected regarding date of stroke, age, years of education, marital status, and employment. Questions on the assessment itself were posed last, with regard to the amount of help participants received during the assessment, the time needed to complete the questionnaire, and the perceived difficulty of the questionnaire.

\section{Data Analysis}

Double and faulty entries were identified by comparing demographic and stroke characteristics and subsequently discarded. Questionnaires not independently completed, were analyzed separately. Of all the correct entries, descriptive statistics were calculated. The association of information status, fatigue severity (VNSF), and fatigue impact (FSS7) with demographic data, age, sex, education, post-stroke time, onset time of fatigue, and nature of PSF was tested using Pearson's correlation coefficient for continuous and $\chi^{2}$-tests for categorical variables. All analyses were conducted using SPSS version 18 for Windows, with Sidak correction for multiple analyses (alpha set at .004).

\section{Results}

Between April 2009 and June 2011 the website drew 9,204 unique visitors. The questionnaire was completed 575 times. Of these 538 could be included, since 33 respondents sent in multiple questionnaires and 4 participants had not had a stroke. Primary analyses were then performed on 479 questionnaires, since 59 entries indicated that they had been filled in with the assistance of another person (50) or had been completed by a proxy (9). 
TABLE 1: Participant characteristics and correlations with VNSF and FSS7 $(N=479)$.

\begin{tabular}{|c|c|c|c|}
\hline & Mean $\pm \mathrm{SD} \%$ & VNSF, $r(P)$ & FSS7, $r(P)$ \\
\hline \multicolumn{4}{|l|}{ Demographic and stroke data } \\
\hline Age (y) & $52.4 \pm 10.8$ & $-.15(<.001)$ & n.s. \\
\hline Time since stroke $(\mathrm{y})$ & $2.73 \pm 4.72$ & n.s. & n.s. \\
\hline Gender male & $45 \%$ & n.s. & n.s. \\
\hline Education (y) & $12.9 \pm 2.5$ & n.s. & n.s. \\
\hline Living with partner & $74 \%$ & n.s. & n.s. \\
\hline Currently employed & $57 \%$ & n.s. & n.s. \\
\hline \multicolumn{4}{|l|}{ Fatigue } \\
\hline Fatigue severity (VNSF) & $6.54 \pm 1.85$ & & $.39(<.001)$ \\
\hline Both VNSF scales $\geq 7$ & $48 \%$ & & \\
\hline Fatigue impact (FSS7) & $5.52 \pm 1.23$ & $.39(<.001)$ & \\
\hline FSS7 $>5$ & $69 \%$ & & \\
\hline PSF (VNSF $\geq 7$ ) since: & & n.s. & n.s. \\
\hline Prestroke & $18 \%$ & & \\
\hline Stroke & $53 \%$ & & \\
\hline $1-6$ months after stroke & $19 \%$ & & \\
\hline$>6$ months after stroke & $10 \%$ & & \\
\hline \multicolumn{4}{|l|}{ Information and needs } \\
\hline Sum of information scores & $5.87 \pm 2.88$ & n.s. & n.s. \\
\hline Information received on $\mathrm{PSF}^{*}$ & & n.s. & n.s. \\
\hline None & $37 \%$ & & \\
\hline Some & $46 \%$ & & \\
\hline Sufficient & $17 \%$ & & \\
\hline Information on self-management* & & n.s. & n.s. \\
\hline None & $50 \%$ & & \\
\hline Some & $37 \%$ & & \\
\hline Sufficient & $13 \%$ & & \\
\hline Information on treatment* & & n.s. & n.s. \\
\hline None & $63 \%$ & & \\
\hline Some & $30 \%$ & & \\
\hline Sufficient & $7 \%$ & & \\
\hline Need for PSF treatment* & & $.34(<.001)$ & $.31(<.001)$ \\
\hline Currently receives treatment & $10 \%$ & & \\
\hline No need & $10 \%$ & & \\
\hline Some & $24 \%$ & & \\
\hline Substantial & $56 \%$ & & \\
\hline
\end{tabular}

${ }^{*}$ Categories are combined to none (none), some (a little and some), and sufficient (sufficient and ample/a great need).

Table 1 list the patients' demographic and clinical characteristics, together with their information status and need for treatment, related to the scores on fatigue severity (VNSF) and fatigue impact (FSS7). Except for younger age, no associations between PSF and demographic variables were found.

Only $17 \%$ of the participants indicated they had received sufficient information about PSF and 7\% felt they had received sufficient information about treatment options. In contrast, $54 \%$ of the respondents expressed a substantial need for treatment. Patients who had no need for treatment (11\%) experienced less severe fatigue (VNSF: $M=5.0 \pm 2.0$ ) and reported less fatigue impact (FSS7: $M=4.8 \pm 1.5$ ) than those currently receiving treatment (VNSF $M=7.1 \pm 1.8$; FSS7 $M=5.6 \pm 1.4$ ) or those expressing some to substantial need (VNSF $M=6.7 \pm 1.7$; FSS7 $=5.5 \pm 1.2, P<.001$ ). The amount of need for treatment was associated with fatigue severity and impact, but not with any demographical or stroke variable (all $P>.004$ ). Information status was not related to any stroke, fatigue, or demographic characteristic (all $P>.004$ ).

The experienced nature of PSF on a physical-tomental dimension differed greatly between participants $\chi^{2}(4)=55.33, P<.001$, with $57 \%$ of the patients expressing both physical and mental fatigue components (Figure 1). No associations were found between the nature of PSF 


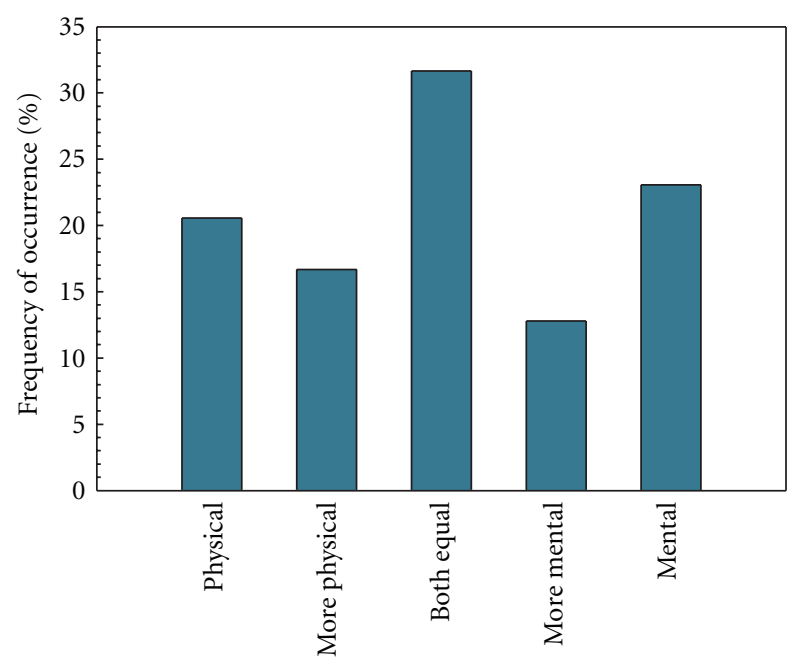

FIGURE 1: Frequency of experienced nature of fatigue $(N=479)$.

and VNSF, FSS7, or information status $\left(\chi^{2}\right.$ tests, all $P>$ $.01)$.

Regarding the questionnaire, $51 \%$ reported having no difficulty at all completing the tool, $30 \%$ a little difficulty, $14 \%$ some, and $5 \%$ had substantial difficulties with filling in the questionnaire. For most participants (53\%) completing the questionnaire took less than 5 minutes, $37 \%$ needed 5 10 minutes, $8 \%$ needed $11-20$ minutes, and only $2 \%$ needed more than 21 minutes.

Questionnaires that were completed with the help of others or by proxies differed from those completed independently on several accounts. Respondents requiring help were older ( $61.3 \pm 1.8$ years), less likely to be employed (20\%), experienced more severe fatigue (VNSF $M=7.7 \pm 1.6$ ), took longer to fill in the questionnaire (Median 6 to 10 minutes), and reported more difficulty filling in the questionnaire.

\section{Discussion}

This internet-based study was conducted to evaluate the need for information and treatment concerning PSF in community-dwelling patients with stroke in The Netherlands. The results indicate that fatigue is still a neglected symptom after stroke, because most of the participants did not receive sufficient information about (the causes) of fatigue $(83 \%)$, about self-management techniques (87\%) or other treatment options (93\%). This worrisome lack of information shows a remarkable contrast with the fact that $54 \%$ of participants indicated a substantial need for treatment for PSF.

A large proportion of the participants in the present study experienced severe fatigue as assessed with the VNSF $(48 \%)$ and reported a severe fatigue impact as assessed with the FSS7 $(69 \%)$. The moderate association between both scales reveals that different aspects of PSF were measured. Interestingly, fatigue scores were neither associated with sex or post-stroke time, nor with the onset time or nature of PSF. Only a small negative correlation with age was found. However, patients who did not complete the questionnaire independently were more severely fatigued and older than those filling in the questionnaires without assistance. These results emphasize the persistence of fatigue complaints after stroke, irrespective of when or by whom they are reported.

Most participants experienced fatigue both physically and mentally, which underscores the notion that PSF includes both components. The finding that the need for treatment was associated with fatigue severity and fatigue impact is understandable and emphasizes the importance of providing appropriate information. The result that the need for information itself was not associated with fatigue scores indicates that even patients with relatively mild fatigue should receive adequate information about PSF and its treatment options. It is possible that patients in fact have received information on PSF and treatment options, but due to their mental fatigue were unable to remember the information. In this case, the provision of information should be more adequately tailored to the fatigability and the use of written information such as leaflets could be of aid [35].

This study may have been subject to bias. Most importantly, especially participants with a great need for information and/or need for treatment can be expected to search the internet for information about PSF and its treatment options. As a result, figures about fatigue severity, fatigue impact, and lack of information may have been confounded by selection bias. On the other hand, $63 \%$ of the participants had received at least some information about PSF and 37\% about treatment options. In addition, our respondents were relatively young (52 years of age), highly educated ( $>12$ years), and 57\% still were employed, suggesting preserved cognitive functions, which should have enabled them to receive and ask for appropriate information. Another limitation is that PSF scores may have been influenced by prestroke fatigue or fatigue as a result of concurrent disease(s) occurring after stroke. Yet, prestroke fatigue was reported in only $18 \%$ of the participants with severe fatigue, while $71 \%$ experienced the onset of fatigue within 6 months after stroke.

\section{Conclusion}

PSF is an important clinical symptom after stroke, requiring attention by medical professionals as well as treatment and/or care. It is, however, still a neglected phenomenon in The Netherlands.

\section{Acknowledgment}

The study was funded by The Netherlands Organization of Health Research and Development (ZonMw) Grant 14350053.

\section{References}

[1] M. H. de Groot, S. J. Phillips, and G. A. Eskes, "Fatigue associated with stroke and other neurologic conditions: implications for stroke rehabilitation," Archives of Physical Medicine and Rehabilitation, vol. 84, no. 11, pp. 1714-1720, 2003. 
[2] G. E. Carlsson, A. Möller, and C. Blomstrand, "Consequences of mild stroke in persons < 75 years-a 1-year follow-up," Cerebrovascular Diseases, vol. 16, no. 4, pp. 383-388, 2003.

[3] J. Bogousslavsky, "William Feinberg lecture 2002: emotions, mood, and behavior after stroke," Stroke, vol. 34, no. 4, pp. 1046-1050, 2003.

[4] A. Lerdal, L. Bakken, S. Kouwenhoven et al., "Poststroke fatigue- - review," Journal of Pain and Symptom Management, vol. 38, no. 6, pp. 928-949, 2009.

[5] V. Schepers, A. Visser-Meily, M. Ketelaar, and E. Lindeman, "Poststroke fatigue: course and its relation to personal and stroke-related factors," Archives of Physical Medicine and Rehabilitation, vol. 87, no. 2, pp. 184-188, 2006.

[6] D. Christensen, S. P. Johnsen, T. Watt, I. Harder, M. Kirkevold, and G. Andersen, "Dimensions of post-stroke fatigue: a twoyear follow-up study," Cerebrovascular Diseases, vol. 26, no. 2, pp. 134-141, 2008.

[7] S. P. van der Werf, H. L. van Den Broek, H. W. Anten, and G. Bleijenberg, "Experience of severe fatigue long after stroke and its relation to depressive symptoms and disease characteristics," European Neurology, vol. 45, no. 1, pp. 28-33, 2001.

[8] B. Y. Tseng, S. A. Billinger, B. J. Gajewski, and P. M. Kluding, "Exertion fatigue and chronic fatigue are two distinct constructs in people post-stroke," Stroke, vol. 41, no. 12, pp. 2908 2912, 2010.

[9] H. Naess, H. I. Nyland, L. Thomassen, J. Aarseth, and K. M. Myhr, "Fatigue at long-term follow-up in young adults with cerebral infarction," Cerebrovascular Diseases, vol. 20, no. 4, pp. 245-250, 2005.

[10] I. G. van de Port, G. Kwakkel, V. P. Schepers, C. T. Heinemans, and E. Lindeman, "Is fatigue an independent factor associated with activities of daily living, instrumental activities of daily living and health-related quality of life in chronic stroke?" Cerebrovascular Diseases, vol. 23, no. 1, pp. 40-45, 2007.

[11] M. Bendz, "The first year of rehabilitation after a stroke-from two perspectives," Scandinavian Journal of Caring Sciences, vol. 17, no. 3, pp. 215-222, 2003.

[12] G. E. Mead, C. Graham, P. Dorman et al., "Fatigue after stroke: baseline predictors and influence on survival. analysis of data from UK patients recruited in the international stroke trial," PLoS ONE, vol. 6, no. 3, Article ID e16988, 2011.

[13] E. L. Glader, B. Stegmayr, and K. Asplund, "Poststroke fatigue: a 2-year follow-up study of stroke patients in Sweden," Stroke, vol. 33, no. 5, pp. 1327-1333, 2002.

[14] H. Naess, L. Lunde, J. Brogger, and U. Waje-Andreassen, "Fatigue among stroke patients on long-term follow-up. The Bergen stroke study," Journal of the Neurological Sciences, vol. 312, no. 1-2, pp. 138-141, 2012.

[15] N. A. Flinn and J. E. Stube, "Post-stroke fatigue: qualitative study of three focus groups," Occupational Therapy International, vol. 17, no. 2, pp. 81-91, 2010.

[16] J. H. White, K. R. Gray, P. Magin et al., "Exploring the experience of post-stroke fatigue in community dwelling stroke survivors: a prospective qualitative study," Disability and Rehabilitation, vol. 34, no. 16, pp. 1376-1384, 2012.

[17] E. McGeough, A. Pollock, L. N. Smith et al., "Interventions for post-stroke fatigue," Cochrane Database of Systematic Reviews, no. 3, article CD007030, 2009.

[18] A. M. Zedlitz, T. C. Rietveld, A. C. Geurts, and L. Fasotti, "Cognitive and graded activity training can alleviate persistent fatigue after stroke: a randomized, controlled trial," Stroke, vol. 43, no. 4, pp. 1046-1051, 2012.

[19] F. Duncan, M. A. Kutlubaev, M. S. Dennis, C. Greig, and G. E. Mead, "Fatigue after stroke: a systematic review of associations with impaired physical fitness," International Journal of Stroke, vol. 7, no. 2, pp. 157-162, 2012.

[20] M. A. Kutlubaev, F. H. Duncan, and G. E. Mead, "Biological correlates of post-stroke fatigue: a systematic review," Acta Neurologica Scandinavica, vol. 125, no. 4, pp. 219-227, 2012.

[21] A. Lerdal, L. N. Bakken, E. F. Rasmussen et al., "Physical impairment, depressive symptoms and pre-stroke fatigue are related to fatigue in the acute phase after stroke," Disability and Rehabilitation, vol. 33, no. 4, pp. 334-342, 2011.

[22] A. M. E. E. Zedlitz, J. M. A. Visser-Meily, V. P. Schepers, A. C. H. Geurts, and L. Fasotti, "Patients with severe post-stroke fatigue show a psychosocial profile comparable to patients with other chronic disease: implications for diagnosis and treatment," ISRN Neurology, vol. 2011, Article ID 627081, 8 pages, 2011.

[23] A. Chaudhuri and P. O. Behan, "Fatigue in neurological disorders," The Lancet, vol. 363, no. 9413, pp. 978-988, 2004.

[24] J. Y. Park, M. H. Chun, S. H. Kang, J. A. Lee, B. R. Kim, and M. J. Shin, "Functional outcome in poststroke patients with or without fatigue," American Journal of Physical Medicine and Rehabilitation, vol. 88, no. 7, pp. 554-558, 2009.

[25] D. Claros-Salinas, D. Bratzke, G. Greitemann, N. Nickisch, L. Ochs, and H. Schröter, "Fatigue-related diurnal variations of cognitive performance in multiple sclerosis and stroke patients," Journal of the Neurological Sciences, vol. 295, no. 1-2, pp. 75-81, 2010.

[26] S. Choi-Kwon, J. Choi, S. U. Kwon, D. W. Kang, and J. S. Kim, "Fluoxetine is not effective in the treatment of poststroke fatigue: a double-blind, placebo-controlled study," Cerebrovascular Diseases, vol. 23, no. 2-3, pp. 103-108, 2007.

[27] F. Staub and J. Bogousslavsky, "Fatigue after stroke: a major but neglected issue," Cerebrovascular Diseases, vol. 12, no. 2, pp. 75-81, 2001.

[28] A. W. Barrit and D. G. Smithard, "Targeting fatigue in stroke patients," ISRN Neurology, vol. 2011, Article ID 805646, 6 pages, 2011.

[29] L. Whitehead, "The measurement of fatigue in chronic illness: a systematic review of unidimensional and multidimensional fatigue measures," Journal of Pain and Symptom Management, vol. 37, no. 1, pp. 107-128, 2009.

[30] J. H. Vercoulen, C. M. Swanink, J. F. Fennis, J. M. Galama, J. W. van der Meer, and G. Bleijenberg, "Dimensional assessment of chronic fatigue syndrome," Journal of Psychosomatic Research, vol. 38, no. 5, pp. 383-392, 1994.

[31] L. B. Krupp, N. G. LaRocca, J. Muir-Nash, and A. D. Steinberg, "The fatigue severity scale. Application to patients with multiple sclerosis and systemic lupus erythematosus," Archives of Neurology, vol. 46, no. 10, pp. 1121-1123, 1989.

[32] V. Mathiowetz, K. M. Matuska, and M. E. Murphy, "Efficacy of an energy conservation course for persons with multiple sclerosis," Archives of Physical Medicine and Rehabilitation, vol. 82, no. 4, pp. 449-456, 2001.

[33] H. Gonzalez, T. Olsson, and K. Borg, "Management of postpolio syndrome," The Lancet Neurology, vol. 9, no. 6, pp. 634642, 2010.

[34] C. S. Cleeland, T. R. Mendoza, X. S. Wang et al., "Assessing symptom distress in cancer patients: the M. D. Anderson symptom inventory," Cancer, vol. 89, no. 7, pp. 1634-1646, 2000.

[35] R. P. C. Kessels, "Patients' memory for medical information," Journal of the Royal Society of Medicine, vol. 96, no. 5, pp. 219-222, 2003. 


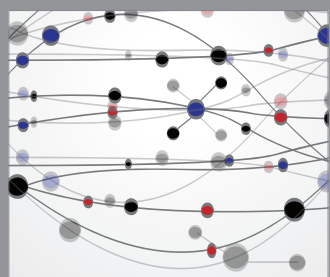

The Scientific World Journal
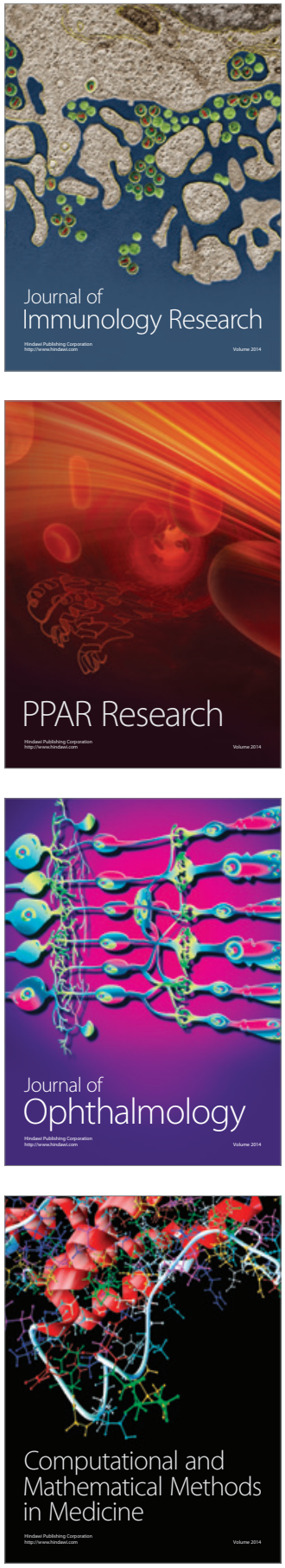

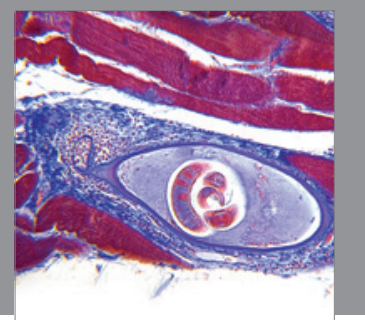

Gastroenterology

Research and Practice
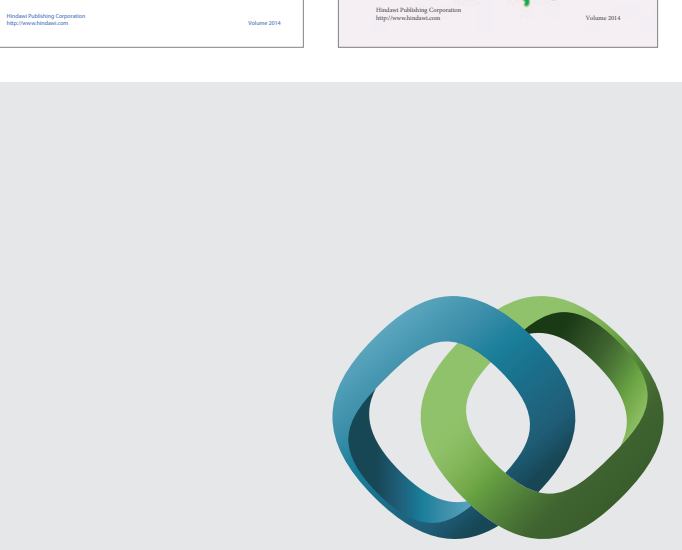

\section{Hindawi}

Submit your manuscripts at

http://www.hindawi.com
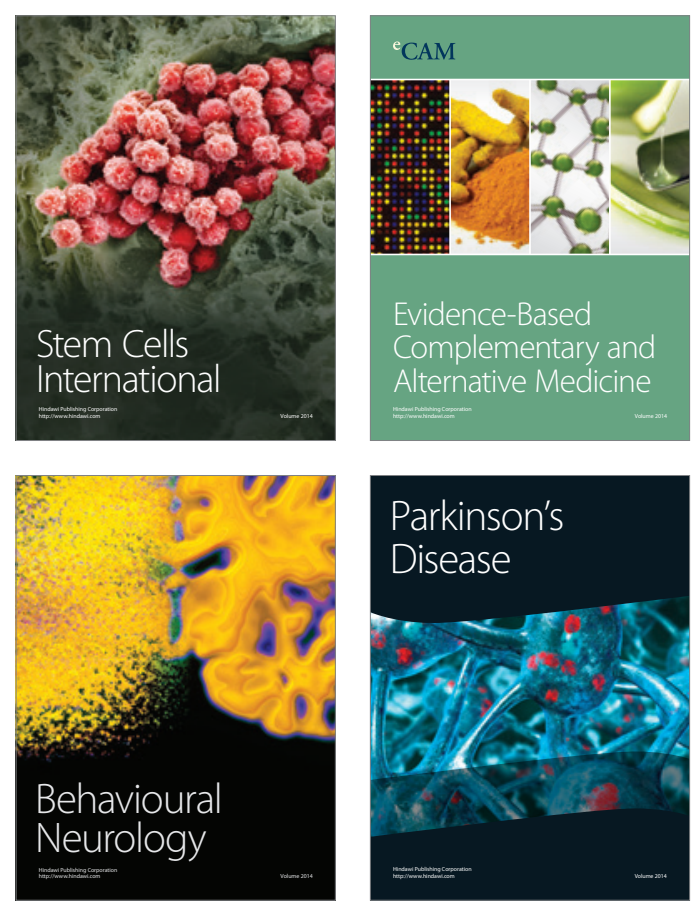

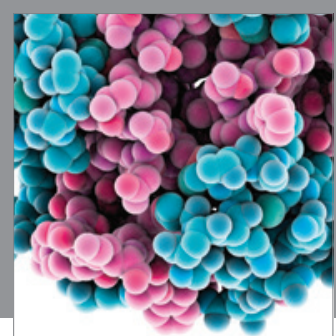

Journal of
Diabetes Research

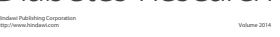

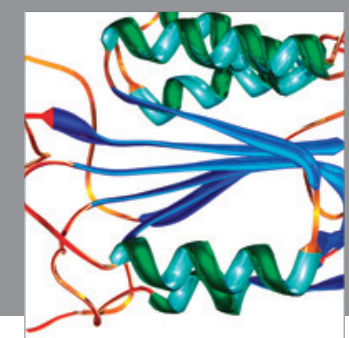

Disease Markers
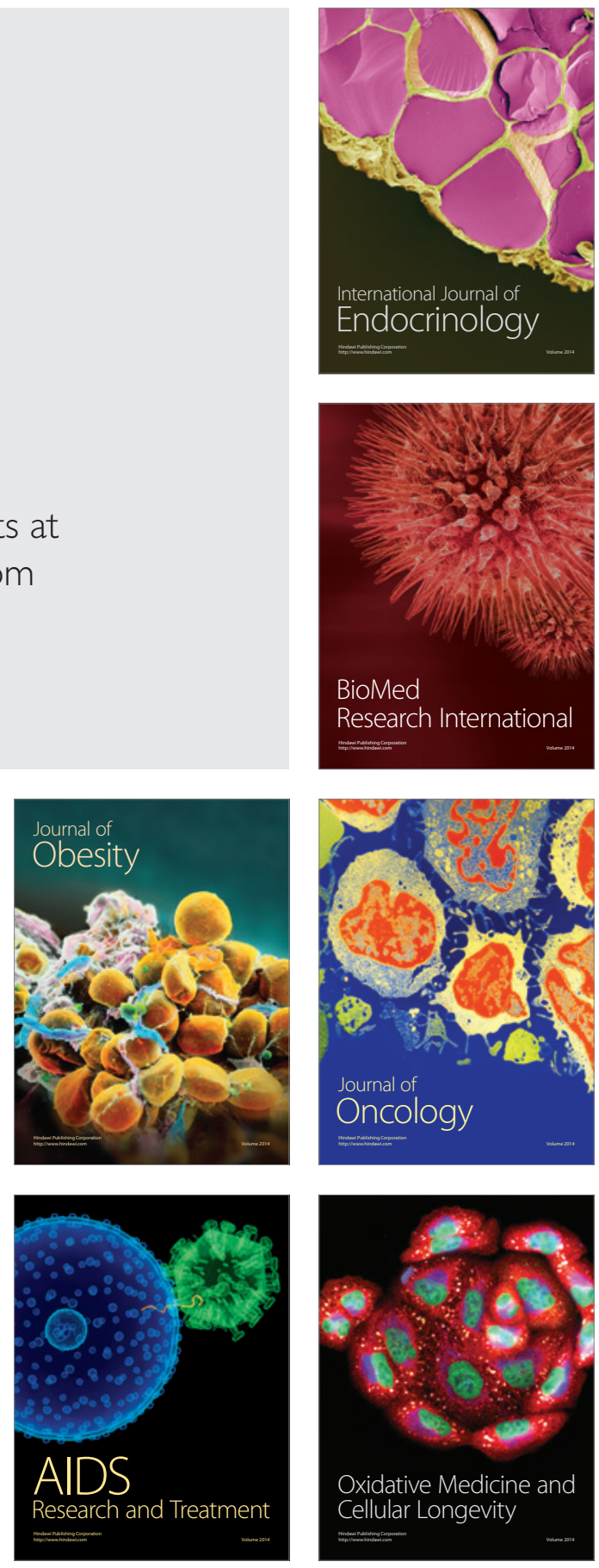